\title{
Estilos e estratégias de aprendizagem em Educação a Distância: diferenças e semelhanças conceituais
}

\author{
Raíssa Bárbara Nunes Moraes Andrade ${ }^{1, *}$, Thaís Zerbini²
}

${ }^{1}$ http://orcid.org/0000-0002-4573-5372/ Faculdade de Filosofia, Ciências e Letras de Ribeirão Preto da Universidade de São Paulo (FFCLRP - USP), Brasil

${ }^{2}$ http://orcid.org/0000-0001-6799-3658 / Faculdade de Filosofia, Ciências e Letras de Ribeirão Preto da Universidade de São Paulo (FFCLRP - USP), Brasil

As ações educacionais à distância são uma estratégia de ensino em Instituições de Ensino Superior (IES) e de Organizações e Trabalho (O\&T). Nesta modalidade, os estilos de aprendizagem fornecem informações para o aperfeiçoamento e a elaboração dos métodos instrucionais. As estratégias de aprendizagem são empregadas em Ambientes Virtuais de Aprendizagem (AVA), enquanto recurso tecnológico que potencializa e amalgama um conjunto de competências e habilidades indispensáveis para o sucesso do aluno. O respectivo estudo objetiva investigar as diferenças e as semelhanças conceituais entre estratégias e estilos de aprendizagem, por meio da validação convergente entre instrumentos que medem tais constructos. Participaram virtualmente do estudo cento e trinta e cinco alunos de uma universidade pública, matriculados em um curso superior à distância. Os resultados indicaram que o conceito de estratégias de aprendizagem, por vezes, se converge com o conceito de estilos de aprendizagem, apresentando baixos índices de correlação em sua maioria. Pesquisas futuras devem buscar promover a revisão dos instrumentos utilizados, a partir das discussões apresentadas nesta investigação.

Palavras-chave: educação a distância, estilos de aprendizagem, estratégias de aprendizagem, instituições de ensino superior, características do estudante.

\section{Learning Styles and Learning Strategies in Distance Education: conceptual differences and similarities}

Abstract

Distance learning is a teaching strategy in higher education institutions (HEIs) as well as organizations and work (O\&W). In this modality of instruction, the learning styles provide information for the improvement of instructional methods. Learning strategies are employed in virtual learning environments (VLEs) as a set of indispensable skills and abilities for student success. The objective of this study was to investigate the conceptual differences and similarities between strategies and learning styles through the convergent validation between instruments that evaluate such constructs. One hundred and thirty five students enrolled in a distance learning higher education course from a public university, taking part in this research online. The results indicated that the concept of learning strategies sometimes converges with the concept of learning styles, presenting low correlation indexes in the most part. Future researche should review the instruments from the discussions put forward in this study.

Keywords: distance education, higher education institutions, learning styles, learning strategies, student characteristics.
Estilos y Estrategias de Aprendizaje en la Educación a Distancia: diferencias y similitudes conceptuales

Resumen

Las acciones educativas a distancia son una estrategia de enseñanza en las Instituciones de Enseñanza Superior (IES), así como en las organizaciones y el trabajo $(\mathrm{O} \& \mathrm{~T})$. En esta modalidad de enseñanza, los estilos de Aprendizaje proporcionan información para la mejora y elaboración de los métodos de enseñanza. Las estrategias de aprendizaje se emplean en entornos virtuales de aprendizaje (EVA) en cuanto recurso tecnológico que potencializa y amalgama un conjunto de habilidades y capacidades indispensables para el éxito del estudiante. Este estudio tuvo como objetivo investigar las diferencias conceptuales y las similitudes entre Estrategias y estilos de Aprendizaje a través de la validación convergente entre instrumentos que evalúan dichas construcciones. Participaron virtualmente del estudio ciento treinta y cinco estudiantes inscritos en enseñanza superior à distancia de una universidad pública participaron en este estudio. Los resultados indicaron que el concepto de estrategias de aprendizaje a veces converge con el concepto de estilos de aprendizaje, con índices de correlación bajos en su mayoría. Pesquisas futuras deben buscar promover la revisión de los instrumentos utilizados, a partir de las discusiones presentadas en esta investigación.

Palabras-clave: educación a distancia, estilos de aprendizaje, estrategias de aprendizaje, instituciones de enseñanza superior; características del estudiante. Trabalho, 20(3), 1150-1156. https://doi.org/10.17652/rpot/2020.3.17947 
As transformações pedagógicas, políticas e tecnológicas que perpassam os vigentes contextos de estudo e trabalho não só resultam em significativas modificações nas práticas e ferramentas utilizadas nas ações educacionais, bem como exigem dos agentes envolvidos - profissionais e estudantes - notável capacidade de atualização profissional e adaptação às novas tecnologias de ensino-aprendizagem (Abbad, Zerbini, \& Souza, 2010). Nesse contexto, os programas de Treinamento, Desenvolvimento e Educação (TD\&E) despertam o interesse científico e social, uma vez que se constituem instrumentais de expressivo valor para ações de formação educacional favorecedoras da aquisição de competências vitais e valorizadas pela sociedade atual (Coelho Jr. \& Borges-Andrade, 2008).

Segundo Borges-Andrade (2006), a área de TD\&E está relacionada ao estudo de ações educacionais que propiciam oportunidades de aprendizagem aos integrantes de uma organização, com o objetivo de obter melhorias em seus desempenhos. Essas ações são definidas como estratégias da empresa para a manutenção da competitividade no mercado através do investimento na qualificação de pessoal e no desenvolvimento de competências compatíveis aos objetivos e metas organizacionais (Abbad et al., 2010). Os eventos de TD\&E promovem inovações constantes, em consonância com as exigências impostas pelo cenário globalizado, evitando, assim, a obsolescência das organizações (Coelho Jr. \& Borges-Andrade, 2008).

Cada vez mais, o as ações educacionais ofertadas a distância tornam-se uma estratégia no contexto de instituições de ensino superior (IES) e de organizações e trabalho (O\&T) (Varanda, Zerbini, \& Abbad, 2010). O termo Educação a Distância (EAD) possui diversas conceitualizações, mas pode ser definido como uma abordagem de ensino-aprendizagem, mediada ou não por mídias tecnológicas, que torna possível a ocorrência de diferentes formas de interação entre professor-aluno e aluno-aluno (Vargas \& Abbad, 2006). Para Mill, Dias-Trindade e Moreira (2019), as características fundamentais da EAD são a separação física entre aluno e professor e a utilização massiva das TIC's como mediadoras da relação ensino-aprendizagem. Devido à presença de características como a facilitação do uso de metodologias flexíveis e adaptadas para indivíduos adultos, a EAD permite que discentes ajustem e conciliem seus estudos com outras atividades, sejam elas profissionais ou pessoais, exercendo, consequentemente, um controle mais intenso dos elementos tempo, espaço e ritmo de estudo (Döös \& Wilhelmson, 2011).

A EAD possibilita uma adaptação constante das metodologias de ensino uma vez que é planejada para atender às necessidades de uma grande variedade de indivíduos com diferentes habilidades, preferências ou estilos de aprendizagem. Investigar os estilos de aprendizagem pode fornecer informações para o aperfeiçoamento dos métodos instrucionais e para mudanças de comportamento de quem aprende (Bjork, Dunlosky, \& Kornell, 2013; Martins \& Zerbini, 2016).

Há muitas definições e abordagens teóricas relacionadas aos estilos de aprendizagem, porém, poucas se encontram na área de Psicologia Organizacional e do Trabalho (POT). Ou seja, a área carece de mais estudo sobre essa variável. Para Moraes e Zerbini (2018), estilos de aprendizagem são preferências do aluno relacionadas ao contexto e ambiente de estudo (luz, ruído, etc.), à maneira como sua aprendizagem é regulada (orientação dos professores/tutores em relação a maneira de estudar) e à sua interação interpessoal (estudar, fazer atividades e trocar informações com colegas do curso).

Para Vermunt (1998), estilos de aprendizagem consistem em um conjunto coerente de atividades de aprendizagem que os indivíduos utilizam frequentemente, de acordo com a orientação e as concepções individuais de aprendizagem. Brant (2014), em seu estudo, faz uso da definição de Berings, Poell e Simons (2005), que definem os estilos de aprendizagem como uma tendência a usar uma determinada combinação de atividades para aprender que deriva de uma combinação de preferências e capacidades percebidas.

Nota-se que, de uma maneira geral, os autores analisados consideram os estilos de aprendizagem preferências relacionadas à sua maneira de estudar, que por sua vez, são influenciadas pelas percepções pessoais do aprendiz em relação a seu processo de aprendizagem. A principal semelhança entre as definições está no fato de os estilos de aprendizagem advirem do resultado da reflexão ou percepção do aluno acerca de sua própria aprendizagem. Por outro lado, alguns autores destacam o contexto de estudo e a interação interpessoal dos aprendizes (Moraes \& Zerbini, 2018), outros destacam mais as atividades realizadas no momento de estudo (Berings, Poell, \& Simons, 2005; Vermunt, 1998).

Brant (2014) afirma que há algumas pesquisas estrangeiras evidenciando a influência dos estilos de aprendizagem em resultados finais de treinamento a distância. Contudo, a análise da produção científica nacional em estilos de aprendizagem mostra que predominam fortemente os estudos em IES. As pesquisas sobre estilos de aprendizagem voltadas para o contexto do trabalho são raras e, em sua maioria, apresentam inconsistência na descrição do método e na apresentação de resultados (Salles, 2007).

No estudo realizado por Brant (2014), notou-se que, praticamente todos os instrumentos que se propõem a medir estilos de aprendizagem possuem problemas relacionados à validade $\mathrm{e}$ a fidedignidade, o que pode levar a uma mensuração não adequada dessa variável. Em alguns outros, há algumas lacunas referentes à própria definição de estilos de aprendizagem. Ganha destaque o instrumento Index of Learnings Styles (ILS) de Vermunt (1998), por ser um instrumento aplicado no contexto de IES em $\mathrm{EaD}$ com melhores índices de validade. Nota-se uma frágil base teórica na maioria dos estudos, o que pode influenciar na qualidade das medidas. A autora destaca a escassez de pesquisas sobre o tema, o que dificulta o desenvolvimento e adaptação das medidas já existentes, bem como propostas de novas medidas.

Os estilos de aprendizagem são considerados características da clientela importantes para a avaliação de necessidades, planejamento, execução e avaliação de treinamentos. Juntamente com os estilos de aprendizagem, as estratégias de aprendizagem, também incluídas em modelos investigação de treinamentos como parte das características dos cursistas, mostra-se de grande valia, influenciando na efetividade dessa ações educacionais (Zerbini \& Abbad, 2010).

Estratégias de aprendizagem são procedimentos (habilidades cognitivas e comportamentais) utilizados pelos indivíduos durante as atividades de aprendizagem, de modo a garantir o sucesso de todas as etapas do processo. São procedimentos focados apenas em atividades de apren9dizagem. As estratégias podem ser modificadas por treinamento com o intuito de aumentar a efetividade da aprendizagem em uma atividade ou ambiente específico (Zerbini \& Abbad, 2008).

Estratégias de aprendizagem podem ser entendidas como sendo ações sistematizadas pelos aprendizes intentando cumprir determinadas tarefas e alcançar metas estabelecidas. Oferecendo subsídios ao processamento de novos conhecimentos, as estratégias de aprendizagem encontram-se positivamente relacionadas com a aprendizagem exitosa, segundo trabalhos da psicologia organizacional ou do campo educacional que focalizam as características próprias do estudante (Martins, Zerbini, \& Medina, 2018 ; Oliveira, Boruchovitch, \& Santos, 2009).

A discussão sobre as estratégias de aprendizagem empregadas em Ambientes Virtuais de aprendizagem (AVA), enquanto um conjunto de competências e habilidades indispensáveis para a sobrevivência na sociedade cibernética, é examinada por diversos autores. 
Tanto o estudo quanto a inovação no ensino e na aprendizagem de estratégias de aprendizagem em cenários virtuais compõem um terreno a ser pesquisado, que deve considerar os atuais perfis e capacidades dos agentes envolvidos, e os novos contextos, instrumentais e objetivos estabelecidos (Martins \& Zerbini, 2016; Umekawa \& Zerbini, 2015; Zerbini, Abbad, Mourão, \& Martins, 2015).

Martins e Zerbini (2016) testaram um modelo de avaliação de ações educacionais a distância composto por variáveis individuais e de reações, visando identificar quais apresentavam maior poder preditivo e mensurar aquelas com contribuições significativas na explicação da variável critério: aprendizagem. As variáveis que apresentaram relação de predição significativa com aprendizagem foram as estratégias de aprendizagem autorregulatórias, as reações aos procedimentos instrucionais, os acessos ao AVA e a troca de mensagens. Segundo as autoras, a variável estratégias autorregulatórias (parte integrante das estratégias de aprendizagem) contribuiu para a explicação da ocorrência de aprendizagem, apontando que o controle da motivação, ansiedade e atenção pelos alunos no momento de estudo, junto à avaliação do próprio processo de aquisição de aprendizagem (monitoramento da compreensão) durante o decorrer das disciplinas, tiveram influência sobre os resultados nas avaliações de aprendizagem.

O breve apanhado feito por Umekawa e Zerbini (2015) sobre obras dedicadas à variável estratégias de aprendizagem revela a preocupação em compreender as habilidades empregadas pelos alunos a fim de estudarem e aprenderem em diversificadas situações de ensino. As autoras observaram uma atenção voltada para o fomento das estratégias de aprendizagem metacognitivas (processos realizados pelos sujeito de forma consciente e que lhe permite ponderar seu próprio pensar, tal como a proposição de objetivos para o estudo e o monitoramento da própria compreensão) e ligadas à gestão do tempo - tidas como muito relevantes para os processos de autorregulação do conhecimento e do tempo, tão presentes nos AVAs. De acordo com Beluce e Oliveira (2012), o campo das produções científicas voltado ao estudo das estratégias de aprendizagem em contextos de ensino online vem testemunhando avanços satisfatórios, porém é necessário que as investigações continuem acontecendo e se aprofundando gradativamente, tendo em vista a seriedade do tema à educação, presencial e a distância. Sendo assim, faz-se indispensável o desenvolvimento de pesquisas que não somente se detenham nos aspectos já considerados, mas lancem mão de novas contribuições ao estudo desta temática.

Warr e Allan (1998) atribuem a mesma importância para o estudo de estilos e estratégias de aprendizagem, já que informações sobre preferências do indivíduo (estilos) podem ser úteis para adaptar os procedimentos utilizados no evento instrucional. Porém, destacam a importância de diferenciar esses dois conceitos. Tendo como base a apreciação das questões empíricas que permeiam o campo literário nacional e estrangeiro em TD\&E, com foco no subsistema de avaliação de treinamento e em agendas de pesquisas de investigações anteriores que se voltaram a essa temática, o problema de pesquisa desse estudo foi delimitado.

A partir da revisão de literatura, percebe-se que os estilos de aprendizagem estão sendo pouco estudados na área de POT, sua conceituação não está bem definida, e os instrumentos que se propõem a medir o construto não possuem evidências de validade. Além disso, por mais que alguma vezes seja estudado em conjunto com as estratégias de aprendizagem, as diferenças conceituais entre os construtos não estão traçadas claramente.

Os autores Morales Vallejo, Sanz, \& Blanco (2003), ao discorrerem a respeito das evidências de validade de instrumentos, demonstram que a análise convergente pode ser utilizada para provar as hipóteses elaboradas em relação ao construto estudado. Ou seja, ela é um meio para verificar relações estabelecidas com variáveis medidas por outros instrumentos que intencionalmente medem o mesmo construto, bem como relações com instrumentos que medem outros aspectos com o qual se espera que exista uma relação positiva ou negativa.

Dessa maneira, no processo de análise convergente é necessário especificar as hipóteses previstas entre as variáveis envolvidas indicando: 1. O sentido que se espera da relação, seja positivo, negativo, ou ausência de relação; e, 2. A magnitude relativa esperada da relação, onde se pode comprovar a existência de relações maiores e mais claras (Silva, Macêdo \& Silva, 2013). No caso deste estudo, a análise convergente irá contribuir para verificar a relação entre os instrumentos de estilos de aprendizagem e estratégias de aprendizagem: quais fatores possuem correlação positiva entre si, indicando que provavelmente estão medindo o mesmo aspecto. Os fatores que não se correlacionarem indicarão diferenças conceituais entre os construtos, pois medirão aspectos diferentes. Em função disso, propõem-se o seguinte problema de pesquisa: Quais as diferenças e as semelhanças conceituais entre estratégias de aprendizagem e estilos de aprendizagem?

A presente pesquisa objetivou investigar as diferenças e as semelhanças conceituais entre estratégias de aprendizagem e estilos de aprendizagem, por meio da análise convergente entre os instrumentos que medem tais construtos.

\section{Método}

A pesquisa foi realizada no curso denominado "Licenciatura em Ciências" da Universidade de São Paulo (USP). O curso é semipresencial, visa satisfazer uma demanda de aperfeiçoamento de professores do Ensino Fundamental e Médio paulistas, e está incluso no Programa Universidade Virtual do Estado de São Paulo (UNIVESP). Destina-se ao preparo de indivíduos que desejam se tornar aptos à prática do magistério no Ensino Fundamental ou de docentes atuantes na Educação Básica.

De acordo com o site do curso (www.licenciaturaciencias.usp.br), todo ano são disponibilizadas 360 vagas anuais, que são distribuídas pelos 7 pólos da UNIVESP. No pólo de Ribeirão Preto, no qual foi realizada a coleta de dados, soma-se aproximadamente 205 vagas, considerando os quatro anos de curso. Informações sobre Evasão não foram repassadas. Dessa maneira, não se pode afirmar com exatidão a quantidade de pessoas formadas ou que estão realizando o curso.

\section{Participantes}

A amostra foi composta por 135 respondentes. Se considerarmos o total de 205 vagas, o índice de retorno foi de $65,8 \%$. Os dados dos respondentes mostram que a maioria dos alunos é do sexo feminino $(57,8 \%)$, casada $(48,9 \%)$ e com filhos $(58,5 \%)$. A idade mais frequente entre os participantes é entre 40 e 49 anos (30,4\%), possuindo, em média, 38 anos de idade $(D P=10,16)$. A escolaridade mais frequente dos alunos é o Ensino Superior Incompleto (38,5\%). Dentre as profissões apresentadas pelos participantes, a de professor(a), foi a mais frequente $(34,8)$. Por fim, a maioria afirmou ter experiência anterior no uso da internet $(94,8 \%)$.

\section{Instrumentos}

Para o presente estudo, foram escolhidos os instrumentos de estratégias de aprendizagem de Zerbini e Martins (2016) e o instrumento de estilos de aprendizagem de Moraes e Zerbini (2018). O instrumentos foram escolhidos pelo fato de ambos terem sido adaptados para adaptá-la a cenários educacionais de nível superior ofertado a distância. 
Instrumentos utilizados na pesquisa

\begin{tabular}{|c|c|c|c|c|}
\hline Instrumento & Escalas Obtidas & Itens & Alfa & $\begin{array}{l}\text { Cargas } \\
\text { Fatoriais }\end{array}$ \\
\hline \multirow{8}{*}{$\begin{array}{l}\text { Estilos de aprendizagem } \\
\text { (Moraes \& Zerbini, 2018) }\end{array}$} & \multirow{2}{*}{ Ambiente de Estudo } & Realizar meus estudos em lugares bem iluminados. & \multirow{2}{*}{0,76} & 0,61 \\
\hline & & Estudar em ambientes com pouco barulho. & & 0,86 \\
\hline & \multirow{4}{*}{ Regulação Externa } & Receber instruções detalhadas sobre as atividades a serem realizadas no curso. & \multirow{4}{*}{0,63} & 0,39 \\
\hline & & $\begin{array}{l}\text { Que os professores/tutores expliquem claramente o que é importante estudar para } \\
\text { alcançar os objetivos propostos no curso. }\end{array}$ & & 0,48 \\
\hline & & Ter o material impresso para ler. & & 0,44 \\
\hline & & Ser lembrado sobre os prazos de entrega das atividades do curso. & & 0,85 \\
\hline & \multirow[b]{2}{*}{ Interação Interpessoal } & Estudar juntamente com outros alunos. & \multirow[b]{2}{*}{0,40} & 0,84 \\
\hline & & $\begin{array}{l}\text { Participar das atividades de interação virtual (fóruns, bate-papo) para discutir os conteú- } \\
\text { dos com os outros estudantes. }\end{array}$ & & 0,31 \\
\hline \multirow{24}{*}{$\begin{array}{l}\text { Estratégias de aprendiza- } \\
\text { gem (Martins \& Zerbini, } \\
\text { 2016) }\end{array}$} & \multirow{11}{*}{$\begin{array}{l}\text { Estratégias } \\
\text { Autorregulatórias }\end{array}$} & Esforcei-me mais quando percebi que estava perdendo a concentração. & \multirow{11}{*}{0,90} & 0,57 \\
\hline & & Forcei-me a manter a atenção nos estudos quando me senti desinteressado. & & 0,63 \\
\hline & & Esforcei-me mais quando percebi que estava perdendo o interesse no assunto. & & 0,55 \\
\hline & & Revisei a matéria para verificar o quanto eu dominava o conteúdo. & & 0,44 \\
\hline & & Esforcei-me para verificar minha compreensão sobre o que estava sendo ensinado. & & 0,66 \\
\hline & & Busquei solucionar minhas dúvidas ao consultar as apostilas do curso. & & 0,50 \\
\hline & & Busquei compreender melhor os conteúdos ao estudá-los nas apostilas do curso. & & 0,51 \\
\hline & & Fiz anotações sobre o conteúdo do curso. & & 0,47 \\
\hline & & Refleti sobre as implicações que os conteúdos aprendidos poderiam ter. & & 0,81 \\
\hline & & Associei os conteúdos do curso aos meus conhecimentos anteriores. & & 0,40 \\
\hline & & Identifiquei situações diárias em que eu pudesse aplicar os conteúdos do curso. & & 0,61 \\
\hline & \multirow{3}{*}{ Controle da Emoção } & Mantive a calma quando tive dificuldades. & \multirow{3}{*}{0,81} & 0,72 \\
\hline & & Mantive a calma com a possibilidade de ter um rendimento abaixo do esperado. & & 0,78 \\
\hline & & Mantive a calma diante dos erros que cometi ao realizar atividades do curso. & & 0,60 \\
\hline & \multirow{8}{*}{ Estratégias Cognitivas } & Busquei auxílio do tutor para esclarecer minhas dúvidas sobre o conteúdo. & \multirow{8}{*}{0,83} & 0,43 \\
\hline & & Busquei auxílio de colegas nos fóruns para esclarecer minhas dúvidas. & & 0,58 \\
\hline & & Troquei mensagens com os colegas para esclarecer dúvidas sobre o conteúdo do curso. & & 0,44 \\
\hline & & $\begin{array}{l}\text { Tentei entender o conteúdo ao aplicá-lo na prática, ao invés de dedicar tempo lendo ou } \\
\text { pedindo ajuda a alguém. }\end{array}$ & & 0,60 \\
\hline & & Aprendi conteúdos ao mentalizá-los repetidamente até perceber que havia entendido. & & 0,80 \\
\hline & & Repeti mentalmente os conteúdos do curso que gostaria de aprender. & & 0,79 \\
\hline & & Li o conteúdo do curso várias vezes como método para aprender. & & 0,52 \\
\hline & & Fiz esquemas do conteúdo do curso como método para aprender. & & 0,53 \\
\hline & \multirow{2}{*}{$\begin{array}{l}\text { Estratégias } \\
\text { Comportamentais }\end{array}$} & $\begin{array}{l}\text { Busquei outras fontes de pesquisa, fora da internet, relacionadas ao curso para me } \\
\text { ajudar a aprender. }\end{array}$ & \multirow[t]{2}{*}{0,80} & $-0,67$ \\
\hline & & Busquei sites relacionados ao conteúdo do curso para me ajudar a aprender. & & $-0,60$ \\
\hline
\end{tabular}

A Tabela 1 apresenta um resumo dos dados dos instrumentos aplicados no presente estudo, bem como os dados relativos à evidência de validade dos mesmos em estudos anteriores.

O fator 1 do instrumento de estilos de aprendizagem, denominado "ambiente de estudo", apresenta um total de 2 itens que tratam de questões relativas ao ambiente no qual o estudo está sendo realizado, e as preferências do aluno em relação a esse contexto. O fator 2, regulação externa", apresenta um total de 4 itens que descrevem preferências do aluno em ter sua aprendizagem regulada por fatores externos a ele, como por exemplo o material didático e/ou os professores/tutores. O fator 3, "interação interpessoal”, é composto por 2 itens que remetem à preferência do aluno em estudar e/ou realizar as atividades do curso sozinho ou com os demais alunos (Moraes \& Zerbini, 2018)

O fator 1 do instrumento de estratégias de aprendizagem, denominado "estratégias autorregulatórias", apresenta um total de 11 itens relacionados a esforços pessoais para facilitar a aprendizagem. O fator 2, "controle da emoção", apresenta um total de 3 itens que dissertam sobre o controle da ansiedade e prevenção de dispersões de concentração, causadas por sentimentos de ansiedade. O fator 3, "estratégias cognitivas", é composto por 8 itens que falam sobre ajuda interpessoal, aplicação prática, repetição e organização. O fator 4, denominado de "estratégias comportamentais" possui dois itens de busca de ajuda no material didático, que em sua definição fala da obtenção de informações em documentos escritos, manuais de instruções, programas de computador e outras fontes que não envolvam contato social.

Observa-se, a partir da Tabela 1, que os índices de confiabilidade variaram de 0,40 a 0,90 , indicando que as escalas, em sua maioria, apresentam boa consistência interna. É importante destacar que o instrumento de estilos de aprendizagem passou por verificação de evidências de validade pela primeira vez no estudo de Moraes \& Zerbini (2018), o que justifica suas cargas fatoriais um de valores menores que o instrumento de estratégias de aprendizagem, que vem passando por constantes revisões e validações. As cargas fatoriais dos instrumentos, por sua vez, variaram de 0,31 a 0,86 , o que indica que as escalas apresentam validade e contemplam itens representativos do fator.

\section{Procedimentos de Coleta de dados e Cuidados Éticos}

A coleta foi realizada totalmente à distância, por meio da internet, utilizando um aplicativo gratuito, que possibilitou a publicação dos questionários on-line através de um link forneci- 
do aos alunos do curso de Licenciatura em Ciências. As respostas foram gravadas em um software on-line, no formato de planilhas, e posteriormente foram importadas para o SPSS versão 21.0. Somente a pesquisadora responsável teve acesso a esses dados, não sendo, portanto, de domínio público.

O projeto de pesquisa foi submetido ao Comitê de Ética em Pesquisa da Faculdade de Filosofia, Ciências e Letras de Ribeirão Preto (FFCLRP) - USP. Após a aprovação (CAAE n ${ }^{\circ}$ 95854418.5.0000.5407), a universidade participante foi contatada, sendo o coordenador do Pólo de Ribeirão Preto esclarecido sobre os objetivos da pesquisa e os procedimentos que seriam utilizados. Mediante sua autorização, foi possível a realização da pesquisa.

Para que o participante pudesse ter acesso aos questionários e dar início ao preenchimento de suas respostas, era necessário que o mesmo realizasse a leitura e concordasse com Termo de Consentimento Livre e Esclarecido, através do aceite do mesmo na plataforma de coleta de dados on-line.

\section{Procedimentos de Análise de Dados}

Ao realizar a análise convergente, são estudadas as relações esperadas e plausíveis com outras medidas relacionadas. São possíveis dois tipos de relações nesse tipo de validação: 1) Relações estabelecidas com variáveis medidas por outros instrumentos que intencionalmente medem o mesmo construto; e 2) Relações com instrumentos que medem outros aspectos com o qual se espera que exista uma relação positiva ou negativa (Morales Vallejo et al., 2003).

Para a análise convergente entre os instrumentos de estilos de aprendizagem e estratégias de aprendizagem, foram calculados os escores fatoriais dos dois instrumentos, e os mesmos foram submetidos à correlação de Pearson. O objetivo foi verificar o grau e a direção (positiva ou negativa) da correlação entre os escores fatoriais dos instrumentos utilizados. Ou seja, se os mesmos se correlacionam de maneira positiva, negativa, ou simplesmente não se correlacionam significativamente. As correlações positivas tendem a indicar que os escores estão medindo construtos semelhantes, ou seja, indicam semelhanças conceituais entre estilos e estratégias de aprendizagem. As correlações negativas tendem a indicar que os escores estão medindo construtos opostos, ou seja, demonstra diferenças ou características opostas entre estilos e estratégias de aprendizagem. Por fim, a ausência de correlação indica aspectos singulares a um dos construtos.

\section{Resultados}

Foi realizada a análise convergente dos instrumentos utilizados. Para essa análise, como foi citado anteriormente, foi calculada a correlação entre os escores fatoriais dos instrumentos de estilos e estratégias de aprendizagem. $\mathrm{Na}$ Tabela 2 são apresentados os resultados advindos da correlação. Em seguida, os mesmos são relacionados com os dados da literatura, propondo uma maior concepção acerca dos conceitos de estratégias de aprendizagem e estilos de aprendizagem. A Tabela 2 apresenta os resultados da correlação, marcando as correlações significativas para $p<0,01$.

A partir da Tabela 2 pode-se notar que o fator estratégias autorregulatórias se correlacionou positivamente com os fatores ambiente de estudo $(r=0,60 ; p=0)$, regulação externa $(r=0,36 ; p=0)$ e interação interpessoal $(r=0,27 ; p=0)$. Nota-se que sua maior correlação foi apresentada juntamente ao fator ambiente de estudo. $\mathrm{O}$ fator controle da emoção não se correlacionou significativamente com nenhum fator do instrumento de estilos de aprendizagem. O fator estratégias cognitivas também se correlacionou positivamente com os três fatores: ambiente de estudo $(r=0,37 ; p=0)$, regulação externa $(r=0,28 ; p=0)$ e interação interpessoal $(r=0,50 ; p=0)$. A maior correlação desse fator é a apresentada junto ao fator interação interpessoal. Por fim, o fator estratégias comportamentais se correlacionou somente com o fator ambiente de estudo $(r=0,26 ; p=0)$.

A Tabela 2 demonstrou muitas correlações significativas, porém, éimportante destacar que apenas algumas apresentaram índices elevados. Das sete correlações significativas, quatro apresentaram índice superior a 0,30 e dessas, apenas duas foram superiores a 0,50. Sendo assim, por mais que alguns fatores se correlacionem significativamente, eles se correlacionam com índices baixos e medianos.

Os resultados também demonstram que o conceito de estratégias de aprendizagem algumas vezes se converge com o conceito de estilos de aprendizagem, apresentando baixos índices de correlação em sua maioria. Uma hipótese seria que os instrumentos ainda possuem itens com ambos os conceitos, ou seja medindo tanto estilos quanto estratégias de aprendizagem. O fator controle da emoção, por não se correlacionar de maneira alguma com os fatores de estilos de aprendizagem, indica que não possui relação com o conceito de estilos de aprendizagem. Nenhum dos fatores se correlacionaram significativamente de modo negativo entre eles, o que indica que os conceitos de estilos de aprendizagem e estratégias de aprendizagem não possuem definições contrárias entre si.

\section{Discussão}

A validação convergente entre os instrumentos de estratégias de aprendizagem e estilos de aprendizagem demonstrou que as definições desses construtos ainda estão interligadas algumas vezes na literatura, o que traz à tona a necessidade de um maior aprofundamento em relação às diferenças e às semelhanças entre essas duas variáveis. A validade convergente se fez necessária no sentido em que muitos autores tratam de estilos de aprendizagem e estratégias de aprendizagem de uma maneira conjunta, muitas vezes sem separar os dois conceitos, como é o caso de Vermunt (1998), que em seu instrumento Index of Learning Styles adiciona itens que são caracterizados como estratégias de aprendizagem. O fator controle de emoção também é considerado um Estilo de aprendizagem para Vermunt (1998). O autor chama esse fator de estilo autorregulatório, que trata das preferências relacionadas ao controle das emoções durante o estudo, por exemplo: preferir ficar mais calmo ou mais agitado durante os estudos. Porém,

Tabela 2

Correlação entre os fatores dos instrumentos de estilos de aprendizagem e estratégias de aprendizagem

\begin{tabular}{|c|c|c|c|c|c|}
\hline Estratégias de aprendizagem & Correlação & $\begin{array}{c}\text { Estratégias } \\
\text { Autorregulatórias }{ }^{1}\end{array}$ & Controle da emoção ${ }^{1}$ & Estratégias Cognitivas ${ }^{1}$ & $\begin{array}{c}\text { Estratégias } \\
\text { Comportamentais }^{1}\end{array}$ \\
\hline \multirow{2}{*}{ Ambiente de Estudo ${ }^{2}$} & $r$ & $0,60^{*}$ & 0,03 & $0,37^{*}$ & $0,26^{*}$ \\
\hline & $p$ & 0,00 & 0,76 & 0,00 & 0,00 \\
\hline \multirow{2}{*}{ Regulação Externa² } & $r$ & $0,36 *$ & 0,04 & $0,28 *$ & 0,21 \\
\hline & $p$ & 0,00 & 0,66 & 0,00 & 0,02 \\
\hline \multirow{2}{*}{ Interação Interpessoal $^{2}$} & $r$ & $0,27 *$ & $-0,07$ & $0,50 *$ & 0,22 \\
\hline & $p$ & 0,00 & 0,42 & 0,00 & 0,01 \\
\hline
\end{tabular}

Nota. ${ }^{1}$ Fatores do instrumento de estratégias de aprendizagem; ${ }^{2}$ Fatores do instrumento de estilos de aprendizagem; *correlação significativa para $p<0,01$ 
esse fator não se correlacionou com nenhum fator de estilos de aprendizagem, o que sugere que tais itens não são considerados estilos de aprendizagem, não corroborando Vermunt (1998).

No fator de estratégias cognitivas, alguns itens são considerados um estilo de aprendizagem de regulação externa para Brant (2014). Isso pode ter sido um dos motivos pelos quais o fator apresentou uma correlação significativa com o fator de regulação externa. Ainda neste fator, alguns itens tratam de interação do aluno com os demais alunos, o que é considerado por Brant (2014) e Vermunt (1998) um estilo de aprendizagem. Reflexo disso foi o alto índice de correlação do fator estratégias cognitivas com o fator interação interpessoal.

Alguns itens do fator estratégias autorregulatórias são considerados estilos de aprendizagem por Brant (2014), denominado de regulação externa da aprendizagem pelo material didático. $O$ fator apresentou uma correlação significativa com o fator regulação externa do instrumento de estilos de aprendizagem, o que pode indicar uma convergência de conceitos. Não foi encontrada literatura relacionando os itens de estratégias autorregulatórias com os itens de ambiente de estudo que explicasse assim a alta correlação apresentada entre os fatores.

A literatura demonstra que a principal diferença conceitual entre estilos de aprendizagem e estratégias de aprendizagem é que a última é passível de treinamento (Zerbini, Abbad, Mourão, \& Martins, 2015) enquanto que o primeiro, por ser uma preferência individual, não pode ser treinado, mesmo que possa ser alterado de acordo com o contexto de estudo (Vermunt, 1998). A semelhança é que ambos são utilizados para facilitar o processo de aprendizagem. O uso correto das estratégias de aprendizagem e a adaptação dos estudos, segundo os estilos de aprendizagem de cada um, devem auxiliar na aprendizagem. Os resultados empíricos desse estudo demonstraram que, estilos e estratégias de aprendizagem, por não apresentarem correlação negativa, não são conceitos opostos. Possuem semelhanças, uma vez que ambos são características individuais dos cursistas, e algumas diferenças, especialmente no que tange ao fato de que as estratégias são atividades realizadas no momento do estudo, enquanto os estilos são preferências do aluno. Ou seja, os estilos, por utilizar-se da percepção individual de cada aluno sobre o momento do estudo, sobre o curso e sobre si mesmo enquanto aprendiz, demonstra ser uma variável mais interna do indivíduo do que as estratégias de aprendizagem, para as quais o cursista pode ser treinado a fim de melhorar seu desempenho nos estudos, e não depende então de sua percepção sobre a atividade educacional. Cabe, no entanto, realizar mais estudos averiguando as semelhança e a diferença entre essas duas variáveis para que ter mais clareza em que ponto os conceitos se convergem e divergem.

$\mathrm{O}$ instrumento de estilos de aprendizagem utilizado para este estudo possui apenas 8 itens, devendo receber novos, especialmente nos fatores Interação Interpessoal e Ambiente de Estudo que contam somente com dois itens cada um. Deve ser realizada a tentativa de inclusão de novos itens para que seja verificado se o construto estilos de aprendizagem é contemplado somente por esses itens do instrumento ou pode haver outros itens que são abordados pela definição. É importante salientar que a inserção de novos itens deve respeitar a definição do construto.

A principal contribuição deste estudo foi o exame das diferenças e semelhanças conceituais dos estilos e estratégias de aprendizagem, através da análise convergente entre os dois instrumentos. A principal limitação do estudo está relacionada à impossibilidade de generalizar os resultados encontrados na presente pesquisa para outros contextos e participantes, pois foi avaliado apenas um curso, de apenas uma IES.

Os resultados obtidos trazem implicações práticas que referem ao uso das medidas de avaliação de cursos a distância em instituições de ensino e também em organizações de trabalho que oferecem ações educacionais EAD, no que concerne às características dos alunos (estilos e estratégias de aprendizagem). Tendo conhecimento a respeito do melhor método de mensuração dessas variáveis, pode-se compilar informações pertinentes ao aprimoramento do planejamento instrucional adotado e, consequentemente, dos efeitos produzidos. Segundo estudos anteriores (Martins \& Zerbini, 2014; Martins \& Zerbini, 2016; Umekawa \& Zerbini, 2015), o aperfeiçoamento de cursos na modalidade a distância contribui para que a mesma possa ser vista como uma alternativa educacional disponível, com aplicabilidade atestada por meio de pesquisas científicas. Desta maneira, as pesquisas podem fornecer conhecimentos e discussões acerca dos elementos necessários para que a modalidade não caia no descrédito e esteja constantemente à revelia de preconceitos e comentários depreciativos sobre a sua qualidade e eficácia.

Para as próximas pesquisas, sugere-se: 1) Aplicação dos instrumentos de medida utilizados nesta pesquisa em outros contextos e amostras, como por exemplo em organizações que ofereçam cursos a distância; 2) Realizar análises confirmatórias das estruturas empíricas utilizadas nesta pesquisa; 3) Aprofundar as discussões sobre estilos de aprendizagem em contextos de IES e EAD; 4) Realizar modificações visando a complementação e melhoria do instrumento de estilos de aprendizagem, com inserção de novos itens e adaptação de alguns atuais; 5) O uso da Teoria da Resposta ao Item (TRI) para a apresentação de parâmetros de discriminação e dificuldade de cada item dos instrumentos.

\section{Referências}

Abbad, G., Zerbini, T., \& Souza, D. B. L. (2010). Panorama das pesquisas em Educação a Distância no Brasil. Estudos de Psicologia (Natal), 15(3), 291-298. https://doi.org/10.1590/S1413-294X2010000300009

Beluce, A. C., \& Oliveira, K. L. (2012). Ambientes virtuais de aprendizagem: das estratégias de ensino às estratégias de aprendizagem. Em IX ANPED SUL (p. 1-14). Recuperado de http://www.ucs.br/etc/conferencias/index.php/ anpedsul/9anpedsul/paper/viewFile/3006/904

Berings, M. G. M. C., Poell R.F., \& Simons, P. R. J. (2005). Conceptualizing on-the-job learning styles. Human Resource Development Review, 4, 373-400. https://doi.org/10.1177/1534484305281769

Bjork, R. A., Dunlosky, J., \& Kornell, N. (2013). Self-Regulated Learning: Beliefs, Techniques, and Illusions. Annu. Rev. Psychol., 64, 417-44. https://doi. org/10.1146/annurev-psych-113011-143823

Borges-Andrade, J. E. (2006). Avaliação integrada e somativa em TD\&E. Em J. E. Borges-Andrade, G. Abbad, \& L. Mourão (Orgs.), Treinamento, desenvolvimento e educação em organizações e trabalho: fundamentos para a gestão de pessoas (pp. 343358). Porto Alegre: Artmed.

Brant, S. R. C. (2014). Estudo multinivel de antecedentes do impacto no trabalho e da persistência/Evasão em treinamentos a distância (Tese de doutorado, Instituto de Psicologia, Universidade de Brasília). Recuperado de http://repositorio.unb. br/bitstream/10482/15749/1/2014 SandraReginaCorreaBrantPereiradeJesus.pdf

Coelho Jr., F. A., \& Borges-Andrade, J. E. (2008). Uso do conceito de aprendizagem em estudos relacionados ao trabalho e organizações. Paidéia, 18(40), 221-234. https://doi.org/10.1590/S0103-863X2008000200002

Döös, M., \& Wilhelmson, L. (2011). Collective learning: interaction and a shared action arena. Journal of Workplace Learning, 23(8), 487-500. https://doi. org/10.1108/13665621111174852

Martins, L. B., \& Zerbini, T. (2014) Educação a distância em instituições de ensino superior: uma revisão de pesquisas. Revista Psicologia: Organizações e Trabalho, 14(3), p. 271-282. Recuperado de http://pepsic.bvsalud.org/scielo. php? script $=$ sci arttext\&pid $=$ S1984-66572014000300003

Martins, L. B., \& Zerbini, T. (2016). Fatores influentes no desempenho acadêmico de universitários em ações educacionais a distância. Estudos de Psicologia $(\mathrm{Na}$ tal), 21(3), 317-327. https://doi.org/10.5935/1678-4669.20160030

Martins, L. B., Zerbini, T., \& Medina, F. J. (2018). Learning strategies scale: adaptation to Portuguese and factor structure. Revista Psicologia: Reflexão e Crítica, 31(12). https://doi.org/10.1186/s41155-018-0092-1

Mill, D., Dias-Trindade, S., \& Moreira, J. A. (2019). Subsídios para a Educação a Distância como Campo Investigativo. EducaOnline, 13(1), 79-98. Recuperado de https://www.researchgate.net/publication/331963620 Subsidios para a Educacao a Distancia como Campo Investigativo 
Moraes, R. B. N., \& Zerbini, T. (2018). Estilos de aprendizagem em EaD: Construção e evidências de validade de instrumento. Estudos e Pesquisas em Psicologia, 18(1), 149-168. https://doi.org/10.12957/epp.2018.38114

Morales Vallejo, P. M., Sanz, B. U., \& Blanco, A. (2003). Cuadernos de Estadística, Construcción de escalas de actitudes tipo Likert. Madrid: Editorial La Muralla S.A.

Oliveira, K. L., Boruchovitch, E., \& Santos, A. A. A. (2009). Estratégias de aprendizagem e desempenho acadêmico: evidências de validade. Psicologia: teoria e pesquisa, 25(4), 531-536. https://doi.org/10.1590/S0102$\underline{37722009000400008}$

Salles, T. J. (2007). Estilos de aprendizagem no Trabalbo: Análise e Construção de Medidas. (Dissertação de Mestrado, Instituto de Psicologia, Universidade de Brasília, Brasília). Recuperado de http://repositorio.unb.br/bitstream/10482/1985/1/Dissertacao\%20Tatiana\%20J\%20Salles.pdf

Silva, R. P. A., Macêdo, L. C. B., \& Silva, I. L. R. (2013) Avaliação das características psicométricas dos questionários utilizados nos periódicos da área contábil: um estudo longitudinal compreendido no período 2003-2012. XX Congresso Brasileiro de Custos - Uberlândia, MG, Brasil. Recuperado de http:// anaiscbc.emnuvens.com.br/anais/article/viewFile/124/124. Acesso em 28/12/2015

Umekawa, E. E. R., \& Zerbini, T. (2015). Evasão e persistência em ações educacionais a distância: análise do perfil discente. Revista Psicologia: Organizações e Trabalho, 15(2), 188-200. https://doi.org/10.17652/rpot/2015.2.517

Varanda, R. C., Zerbini, T., \& Abbad, A. (2010). Construção e Validação da Escala de Reações à Interface Gráfica para Cursos de Educação a Distância. Psicologia: Teoria e Pesquisa, 26(2), 371-380. https://doi.org/10.1590/S0102$\underline{37722010000200019}$

Vargas, M. R. M. \& Abbad, G. S. (2006). Bases Conceituais em Treinamento, Desenvolvimento e Educação (TD\&E). Em J. E. Borges-Andrade, G. Abbad, \& L. Mourão (Orgs.), Treinamento, desenvolvimento e educação em organizações e trabalho: fundamentos para a gestão de pessoas (pp. 137-158). Porto Alegre: Artmed.

Vermunt, J. D. (1998). The regulation of constructive learning processes. British Journal of Education Psychology, 68, 149-171. https://doi. org/10.1111/j.2044-8279.1998.tb01281.x

Warr, P., \& Allan, C. (1998). Learning strategies and occupational training. Internacional Review of Industrial and Organizational Psychology, 13, 83-121.

Zerbini, T., \& Abbad, G. (2008). Estratégias de aprendizagem em Curso a Distância: Validação de uma Escala. Psico-USF, 13(2), 177-187. https://doi. org/10.1590/S1413-82712008000200005

Zerbini, T., \& Abbad, G. (2010). Aprendizagem induzida pela instrução em contexto de organizações e trabalho: uma análise crítica da literatura. Cadernos de Psicologia Social do Trabalho, 13(2), 177-193.

Zerbini, T., Abbad, G., Mourão, L., Martins, L. B. (2015). Estratégias de aprendizagem em Curso Corporativo a Distância: como Estudamos Trabalhadores? Psicologia, Ciência e Profissão, 35(4), 1024-1041. https://doi.org/10.1590/1982$\underline{3703000312014}$

\section{Informações sobre as autoras:}

\section{Raíssa Bárbara Nunes Moraes Andrade}

Rua Heitor Chiarello, 480. Ap 44. Jardim Irajá. Ribeirão Preto, SP. CEP 14020-520.

E-mail: raissa.nmoraes@gmail.com

\section{Thaís Zerbini}

E-mail: thais.zerbini@gmail.com 